\title{
Enhancement of Fracture for Low-Permeability Reservoir while Drilling using Smart Fluid throughout Experimental Work
}

\author{
Ahmed Z Noah ${ }^{1 *}$, Khalid I Kabel ${ }^{2}$ and Ahmed Gazar ${ }^{2}$ \\ ${ }^{1}$ Egyptian Petroleum Research Institute, Egypt \\ ${ }^{2}$ Department of Petroleum, Faculty of Engineering, American University in Cairo, Egypt
}

\begin{abstract}
Nano-fluids show potential use in applications related to upstream oil and gas industry to improve the performance of several processes such as exploration, drilling and completion, production and enhanced oil recovery operations. However, their applications for water-based drilling mud (WBM) needs attention to address efficient drilling in a High Pressure and High Temperature (HPHT) environment. In the present work, nano-fluid-enhanced WBM (NWBM) are prepared using the nano-fluids of $\mathrm{CuO}$ and $\mathrm{ZnO}$ (size $<50 \mathrm{~nm}$ ) in a xanthan gum aqueous solution as a base fluid and used as an additive in WBM. The nano-fluids are prepared for nano-particle concentrations of 0.1 , 0.3 and $0.5 \%$ of wt. in base. The prepared nano-fluids are added as an additive of $1.0 \%$ (by volume) to WBM. The enhancement in thermal and electrical properties of NWBH is studied. It is observed that NWBM show improved thermal and electrical properties by about $35.0 \%$ compared to WBM. An increased concentration of nanoparticles further enhances electrical and thermal properties of drilling fluids. The NWBM based on $\mathrm{CuO}$ nano-fluid are observed to show improved thermal properties and are more resistant to HPHT condition than ZnO-based NWBM. High pressure rheological studies are conducted on NWBM to understand the effect of nano-fluids on the rheological properties at varying temperatures $\left(25,70,90\right.$ and $\left.110^{\circ} \mathrm{C}\right)$ and pressures $(0.1 \mathrm{MPa}$ and $10 \mathrm{MPa})$. The effect of pressure on the rheology of NWBM is more significant at higher temperatures and indicates that the better rheological stability in case of NWBM. The most significant role that the nano-fluids play is in-stabilizing the viscosity at higher temperatures. The experimental data on flow curves obtained for various NWBM are fitting to the classical drilling fluid rheological models (Power Law model, Bingham Plastic model and Herschel-Bulkley model). The Herschel Bulkley model is observed to be the best fit-model for rheological behavior of NWBM and can be applied for efficient NWBM design.
\end{abstract}

\section{Introduction}

Nano-fluids have recently attracted attention in the petroleum industry as potential solutions to several short comings of conventional drilling fluids [1]. Drilling fluid is in charge of numerous functions during the drilling operation including transport of cuttings to the surface, maintaining the wellbore pressure and stability, lubrication and cooling of the drill bit and isolation of formation fluids by forming a filter cake around the borehole.
Most troubles for some drilling issues are directly or indirectly triggered by fluid related problems including loss of drilling fluid and wellbore instability [2].

Nanotechnology deals with the characterization, production, design and application of materials and devices based on nanometer scale [3]. Similarly, nanoparticles are substances with dimensions in the order of 1 e100 $\mathrm{nm}(\mathrm{nm})$. Nanoparticles have unique properties because their small size and greater surface area per unit volume; thus, they show a more reaction with other molecules. A

${ }^{*}$ Corresponding author: Ahmed Z Noah, Egyptian Petroleum Research Institute, Nasr City, PB 11727 Cairo, Egypt, E-mail: alaanooh@hotmail.com

Accepted: July 21, 2018: Published: July 23, 2018

Copyright: $\odot 2018$ Noah AZ, et al. This is an open-access article distributed under the terms of the Creative Commons Attribution License, which permits unrestricted use, distribution, and reproduction in any medium, provided the original author and source are credited. 
comparison of the surface area to volume ratio of rounded particles of the same material with radius of $1 \mathrm{~mm}, 1$ $\mathrm{mm}$, and $1 \mathrm{~nm}$ was presented by Ref [4].

The nanoparticles are knitted into the structure of materials and bring about reinforcement. In conventional materials, the atoms are located in the interior of the particles; but, for a typical nanomaterial, most of the atoms are located on the surface of the particles. Reactions that produce superb chemical, optical, electrical, thermal, mechanical, magnetic properties etc., occur in nanomaterials [5].

Deposition of chemical vapor involves making nanoparticulate material from the gas phase [6]. The deposition may be direct or by chemical reaction, forming a new product that is different from the volatilized material. In plasma arcing, gas is made to conduct electricity and it gives up its electrons and thus ionizes. The resulting ionized gas is called plasma. This method is applicable in forming carbon nanotubes. Vacuum arcs is a type of plasma discharge [7]. In the process, large droplets are filtered out before deposition [8].

In the past, addition of particles to classical viscoelastic surfactant (VES) fluids was perceived as counterproductive, in that the particles were viewed as pore plugging and thereby damaging to the reservoir permeability and productivity, and their use would diminish the primary reason for using VES fluid. However, it has been found that select nanosized particles can be used to improve the performance properties of VES fluid. The nanoparticle material is an inorganic crystal with very-low solubility by water, oil, or solvent. More recently, it has been discovered that select nanoparticles that exhibit pyroelectric behavior can improve PCM fluid properties further. Typically, pyroelectric particles exhibit an increase in electrostatic charges on their crystal faces when heated. Use of pyroelectric type nanoparticles can give a degree of delayed pseudo cross linking of VES micelles, where fewer intra-micelle associations occur at ambient temperature but a higher amount occurs as the fluid is heated to reservoir temperatures. The pyroelectric-nanoparticle - pseudo cross linked VES fluid developed a pseudo filter cake and was a wall-building fluid, but after the spurt, the leak off control was noticeably lower.

\section{Fluid Viscosity}

Brookfield PVS Rheometer tests were performed to compare classical VES with pyroelectric nanoparticles present at $100 \mathrm{sec}^{-1}$ shear rate. The tests with pyroelectric nanoparticles showed a substantial increase in viscosity. The $1.0 \%$ volume of active VES with pyroelectric nanoparticles showed more viscosity than the classical VES at $2 \mathrm{vol} \%$ active VES. The $2.0 \%$ volume of active VES with pyroelectric nanoparticles showed almost twice the viscosity as classical VES.

\section{Permeabillity Enhancements}

The injection of a regular mud acid (RMA) solution results in dissolution of calcite, clays and feldspar minerals. Consequently, reservoir porosity and permeability can be enhanced in a region extending several meters around the injection well. Nevertheless, high reactivity and a weak flow prevent the penetration of acid in the far field between the wells. This high reactivity also involves the risk of Portier and Vuataz creating wormholes, able to increase the porosity but not always the permeability of the fractured reservoir. Many factors affect mineral dissolution and associated enhancement in formation porosity and permeability, including mineral abundance and distribution in the formation, reaction kinetics, and injection rate.

Finally, this study demonstrated that acid mixtures injection can play a significant role in the development of porosity around injection wells. It was shown by numerical modeling that mixtures of acids have the possibility to react with carbonates and silicates, dissolving them and opening new pores within the reservoir. The positive effect of acid injection on porosity is proportional to the amount of injected acid. Looking for commercial production rates of the wells and other chemical stimulation techniques should be considered such as fracture acidizing. This type of acid job should reach the fracture network of the far field and connect with the injection and production wells.

A new clean biopolymer-based fracturing fluid has been developed. The new fluid has high conductivity, stable viscosity, low pipe friction, excellent proppant transport, and the capability to be used with produced water. This fluid-system development effort was aided by the evolution of a new approach to proppant transport measurement as well as the operational development of a dry-polymer blender used to effectively hydrate the fluid system as part of an on-the-fly treatment. The new fluid has been field-trialed in 14 frac stages over four wells with two million pounds of proppant placed. The application of the new fluid and experience of the field-trials are discussed.

This new clean fluid is widely applicable to fracturing low-permeability reservoirs worldwide and can be used where both water frac and traditional gelled fluids are currently used. This fluid signals a dramatic shift in fracturing low-permeability unconventional reservoirs by coupling excellent proppant transport with high conductivity.

Traditional guar-based fracturing fluids, as commonly used, give poor retained fracture conductivity. This has been shown in several studies in which the effective fracture length is a fraction of the modeled propped fracture length as shown by production history matching [9]. 
Other types of fracturing fluids that have high retained fracture conductivity in tests can be cost prohibitive and often have poor proppant transport, for example, surfactant gels and water fracs, respectively. Another common issue is that the water must be fairly fresh for the fluid chemistry to work properly. A feasibility project was initiated to develop a fracturing fluid that would address the limitations of traditional guar systems. The design objectives of this feasibility project were to produce a fracturing fluid using nanoscale inorganic materials along with polymers under microemulsion to create a smart fluid that is active in nano size and would cause the following:

- Provide good proppant conductivity

- Have sufficient viscosity to created good fracture width

- Provide good proppant transport

- Have good fluid-loss characteristics

- Provide a good viscosity break

- Have stable viscosity at BHT of approximately $200^{\circ} \mathrm{F}$

- Be a simple system to operationally execute

- Be within current economic bounds

- Stay within current HSE guidelines

\section{Procedures}

Hydraulic fracturing is a technique that aims to increase well productivity. The fracturing gel is applied against the reservoir rock under high differential pressure to create fractures. A proppant (sand, bauxite or ceramic) is pumped into the well with the fracturing gel with the purpose of keeping the fracture open and creating a high conductivity path that eases the flow of fluids from the geological formation to the well. A fracturing gel should provide sufficient viscosity to suspend and transport the proppant into the fracture and should break into a low-viscosity fluid after the fracturing is completed. This break facilitates the fracture cleaning by allowing rapid counter flow of fluids to the sur- face. According to Terracina, et al. [9], a broken gel is one that has a final room temperature viscosity less than $8 \mathrm{mPa} \mathrm{s}$ at 511 s_1 on a Fann model 35 for Rheometer. According to Voneiff, et al. [10], a fracturing gel must break to a viscosity of $50 \mathrm{mPa}$ s to ensure that the gas reserves are maximized.

Surfactant-based fracturing gels can be broken when exposed to hydrocarbons or formation water. Therefore, conventional breakers, commonly used in polymer-based gels, are not required, and the oil or gas produced can act as breakers for surfactant-based gels. Current methodologies devised for gel break tests are specific for polymer-based fracturing gels. Therefore, it is necessary to investigate a proper methodology for surfactant-based gels.

\section{Experimental}

\section{Materials and methods:}

Chemicals: The chemicals used to prepare the smart fluid were: A commercial anionic surfactant isoamyl al-

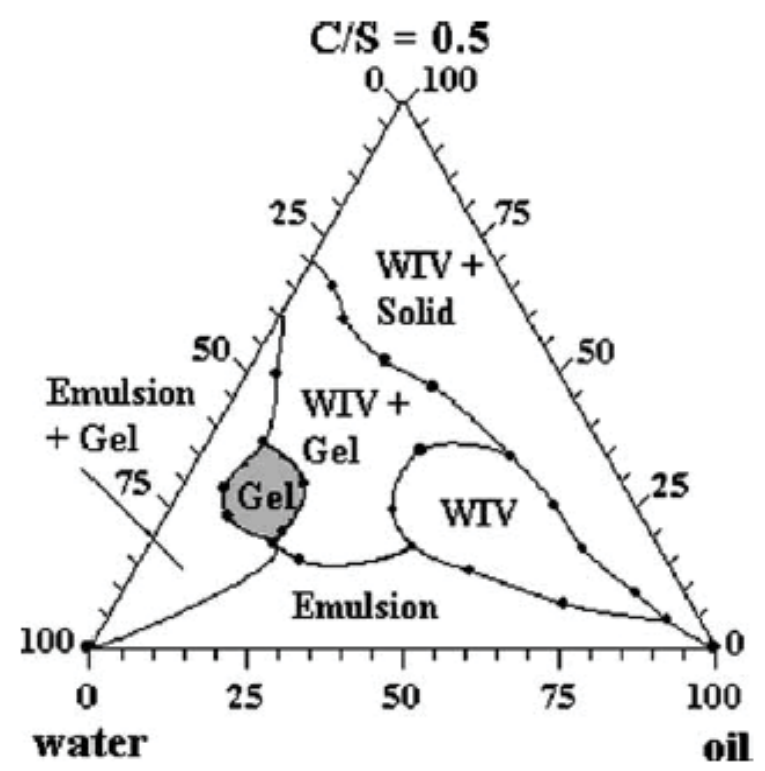

Figure 1: Pseudo-ternary diagram.

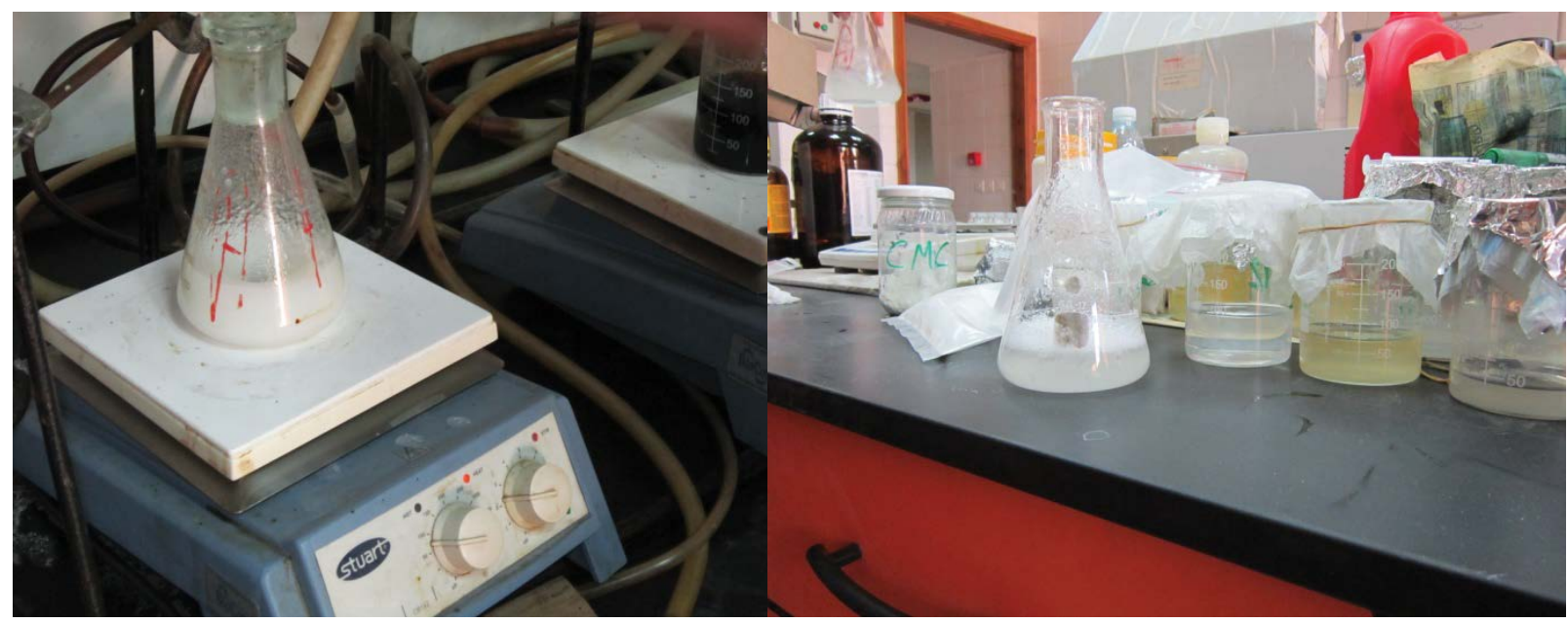

Figure 2: Waring blender. 
cohol; pine oil, CMC, guar gum, distilled water and other chemicals.

Pseudo-ternary phase diagrams: To determine the Winsor's regions in a pseudo-ternary diagram (Figure 1), the oil phase was mixed with the surfactant/co-surfactant pseudo component and the mixture was titrated with water to observe the changes in the Winsor's regions. The volume of water used was determined in each phase change assay. The pseudo-ternary phase diagram was constructed by plotting the amounts of water, oil, and surfactant/co surfactant phases used in the experiment.

Preparation of chemical: After obtaining the pseudo-ternary diagram with a gel region, a point inside the gel region was chosen. The gel was prepared in a Waring Blender, where all the chemicals were added at the same time, and stirred for $30 \mathrm{~min}$ (Figure 2).

Rheological measurements and core samples measurements: Rheological experiments were performed in a Brookfield rheometer. The apparent viscosity measurements were obtained (Table 1 ) by varying shear rate or time, at different temperatures. It was found that, in these experiments, a period of time of $1 \mathrm{~min}$, prior to the actual measurement recording, was necessary in order to allow the system to reach equilibrium (so as to avoid complexities arising from time-dependent phenomena related to gel rheology). Other than both these the samples permeability, porosity, stress, strain and hardness were measured (Table 2).

The experiments were conducted in the following sequence:

1. In order to determine the absolute permeability of the core samples, brine $(2 \mathrm{wt} . \% \mathrm{KCl})$ was injected at a constant flow rate into the core, in the opposite direction to oil production, at $100 \mathrm{psi}$;

2. Once the core sample was $100 \%$ saturated with brine, oil was injected at a constant rate, in the production direction, at 200 psi. The oil injection into the core sample was continued until obtaining connate water saturation;

3. To simulate reservoir conditions, brine was again injected into the core sample at constant rate, once the reservoirs presented residual oil, in the opposite direction to oil production, at $200 \mathrm{psi}$;

4. The fluid was then injected at a constant flow rate, in the opposite direction to oil production, at 200 psi.

Samples were collected, initially with some oil content, until limpid samples were observed. These were collected at specifically determined periods of time, starting from the activation of a chronometer when the pressure was applied. After the samples were collected, gel break was verified by assessing the viscosity, in a Brookfield rheometer, at $268 \mathrm{C}$.

In addition to this the samples were scanned using SEM (Figure 3).

Testing: Basically, the test consisted on the injection of fluids into sandstone core sample and the carbonate core sample (Figure 4) collected from the American university in Cairo core lab. The core samples were isolated in a vacuum chamber. The brine used was composed of $2.0 \%$ wt. of $\mathrm{KCl}$. The crude oil used presented $130 \mathrm{mPa}-\mathrm{s}$ viscosity and $0.89 \mathrm{gm} / \mathrm{cm}^{3}$ density, at $26^{\circ} \mathrm{C}$. All the core samples were roasted at $700{ }^{\circ} \mathrm{C}$ for 18 hours, prior to isolation with resin, with the purpose of removing humidity and increasing core permeability value.

The experiments were conducted in the following sequence:

1. In order to determine the absolute permeability of the core samples, brine $(2.0 \% \mathrm{wt}$. of $\mathrm{KCl})$ was injected at a constant flow rate into the core, in the opposite direction to oil production, at $100 \mathrm{psi}$;

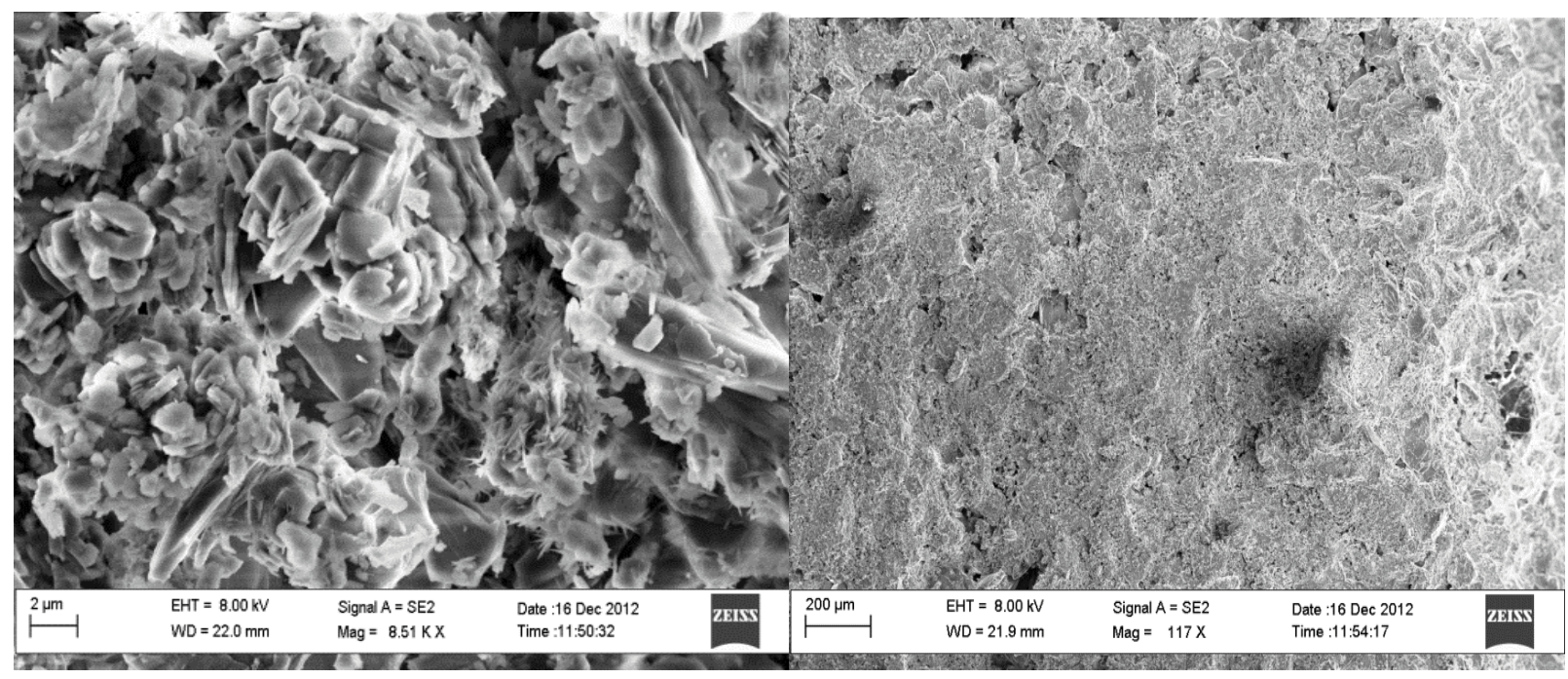

Figure 3: Scan electron microscopy (SEM) for the selected Sandstone sample. 


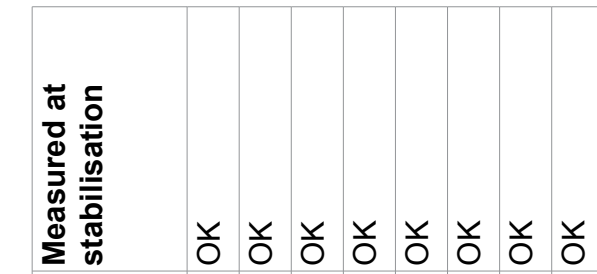

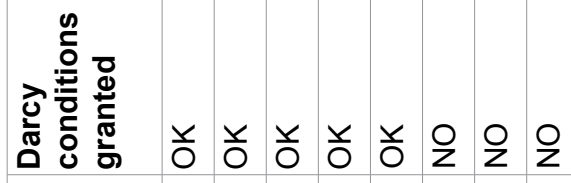

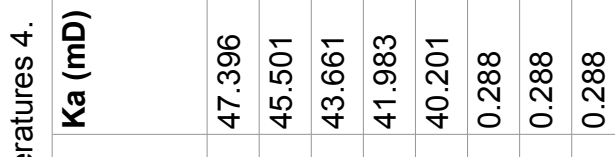
产

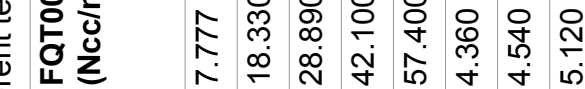

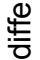

ह.

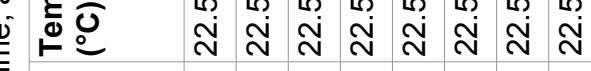

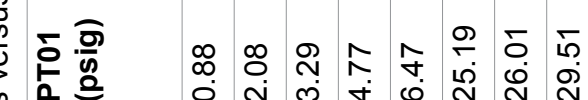

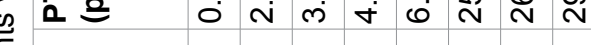

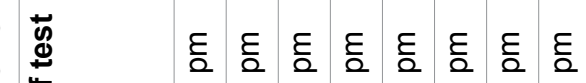

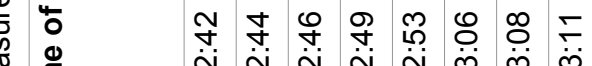

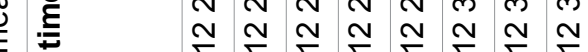

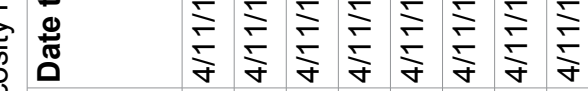

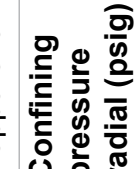
造

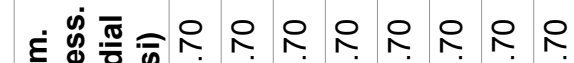

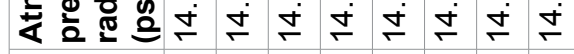

苟 ఏ है

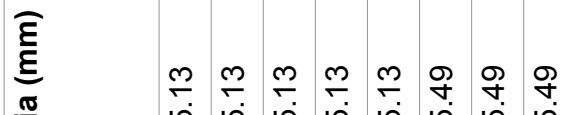

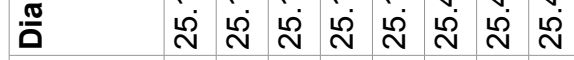

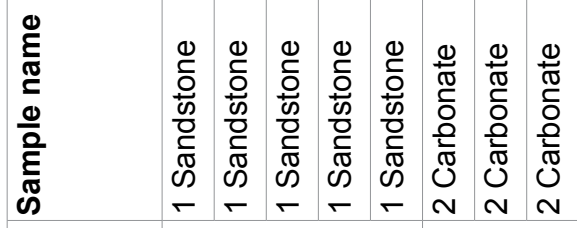
$\frac{0}{\frac{0}{0}}$
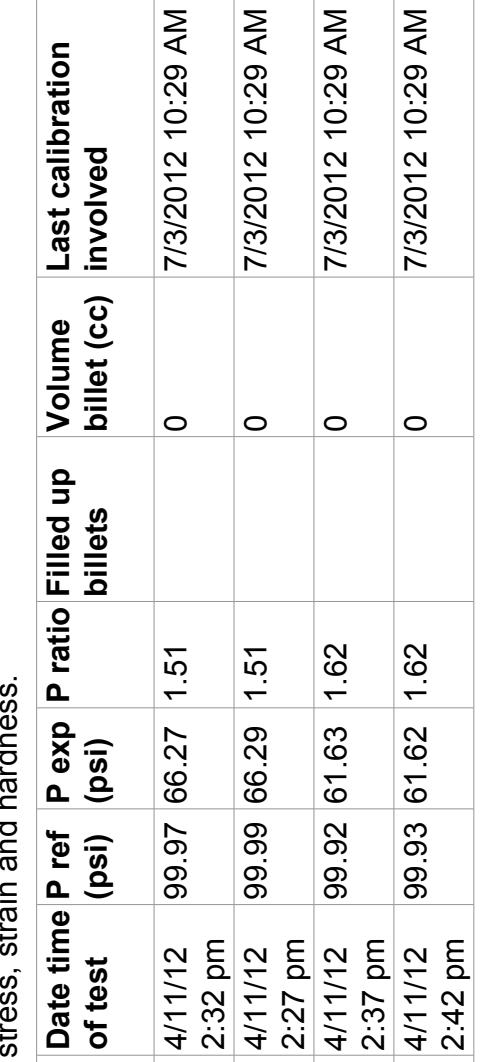

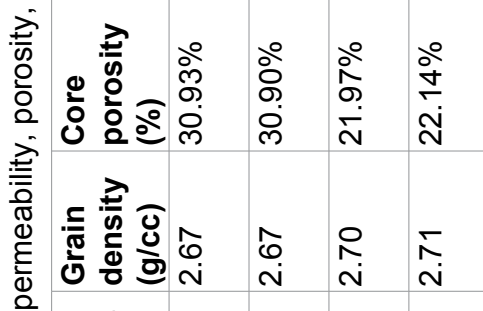

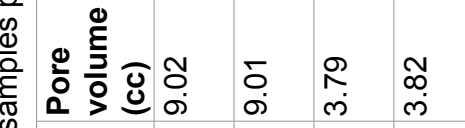

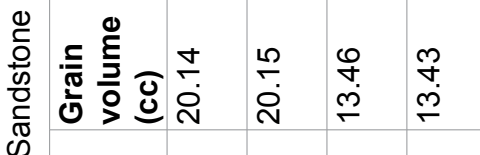
ஸ்

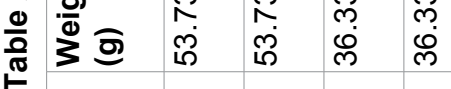

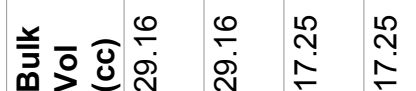
응 ᄃ 0 -

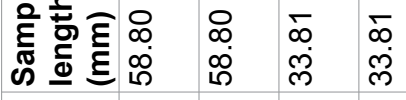

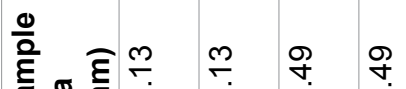

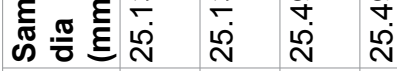

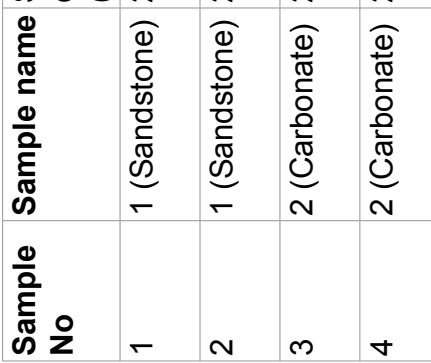


2. Once the core sample was $100 \%$ saturated with brine, oil was injected at a constant rate, in the production

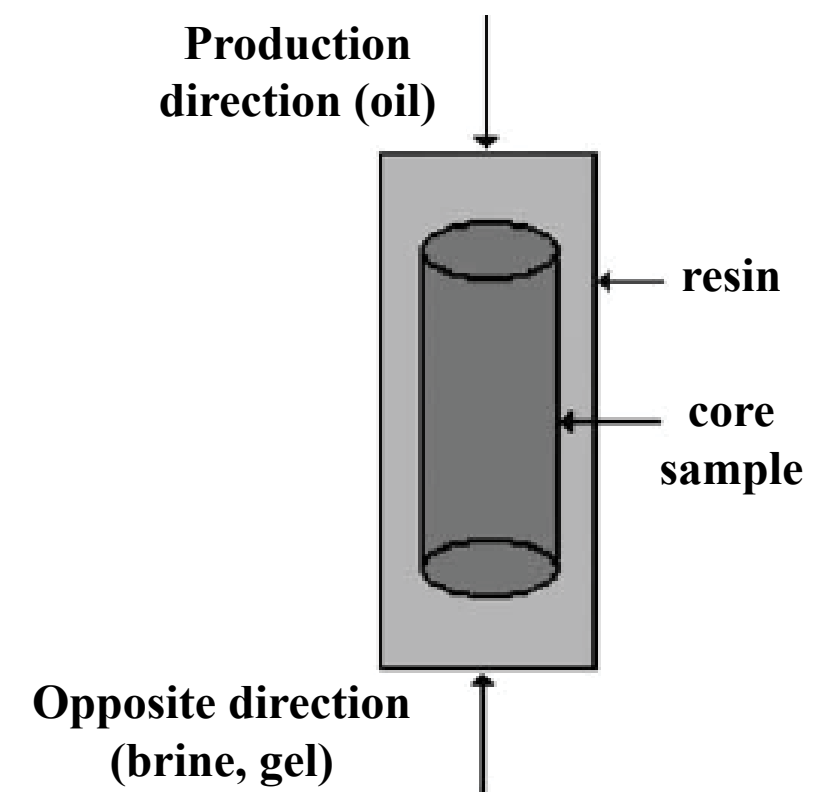

Figure 4: Schematic diagram for the core sample. direction, at 200 psi. The oil injection into the core sample was continued until obtaining connate water saturation (Swi);

3. To simulate reservoir conditions, brine was again injected into the core sample at constant rate, once the reservoirs presented residual oil, in the opposite direction to oil production, at $200 \mathrm{psi}$; and

4. The fluid was then injected at a constant flow rate, in the opposite direction to oil production, at $200 \mathrm{psi}$.

Samples were collected, initially with some oil content, until limpid samples were observed. These were collected at specifically determined periods of time, starting from the activation of a chronometer when the pressure was applied. After the samples were collected, gel break was verified by assessing the viscosity, in a Brookfield Rheometer, at $26^{\circ} \mathrm{C}$.

\section{Results and Discussion}

\section{Pseudo-ternary phase diagram}

A pseudo-ternary phase diagram was constructed in order to identify the gel region. A well-defined gel region

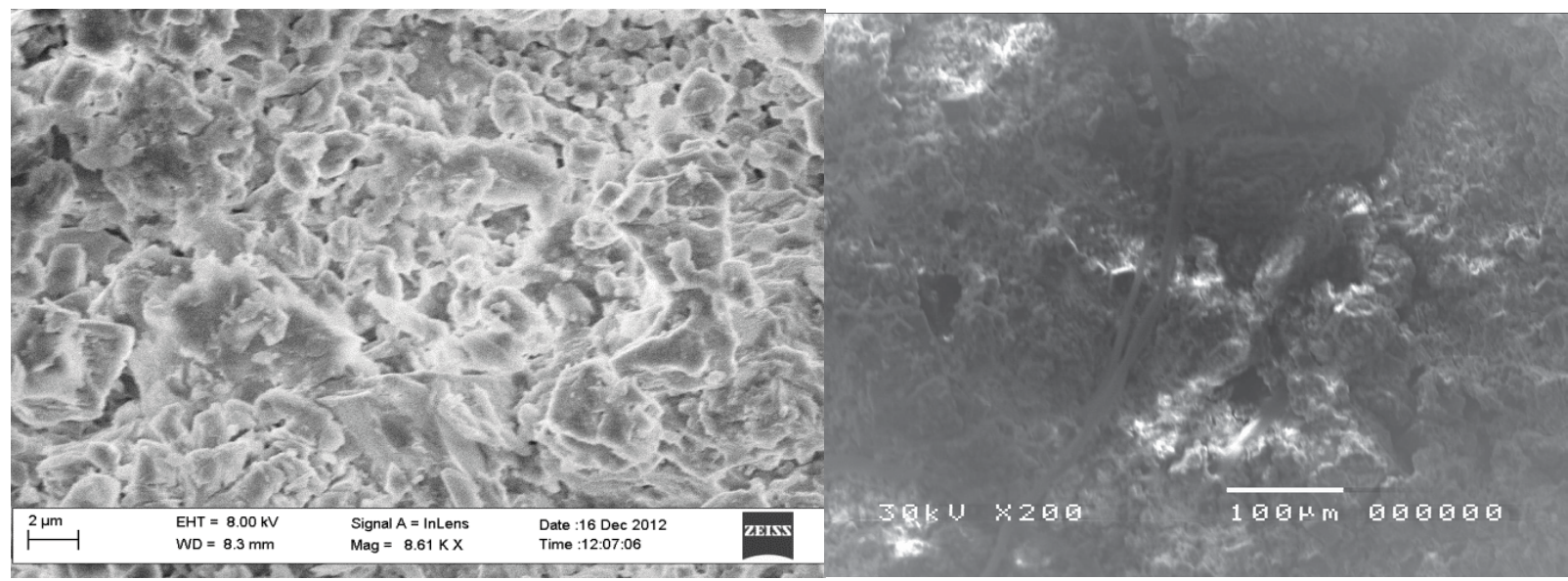

a) Before injection

b) After injection

Figure 5: a) Carbonate sample before injection; b) Carbonate sample after injection.

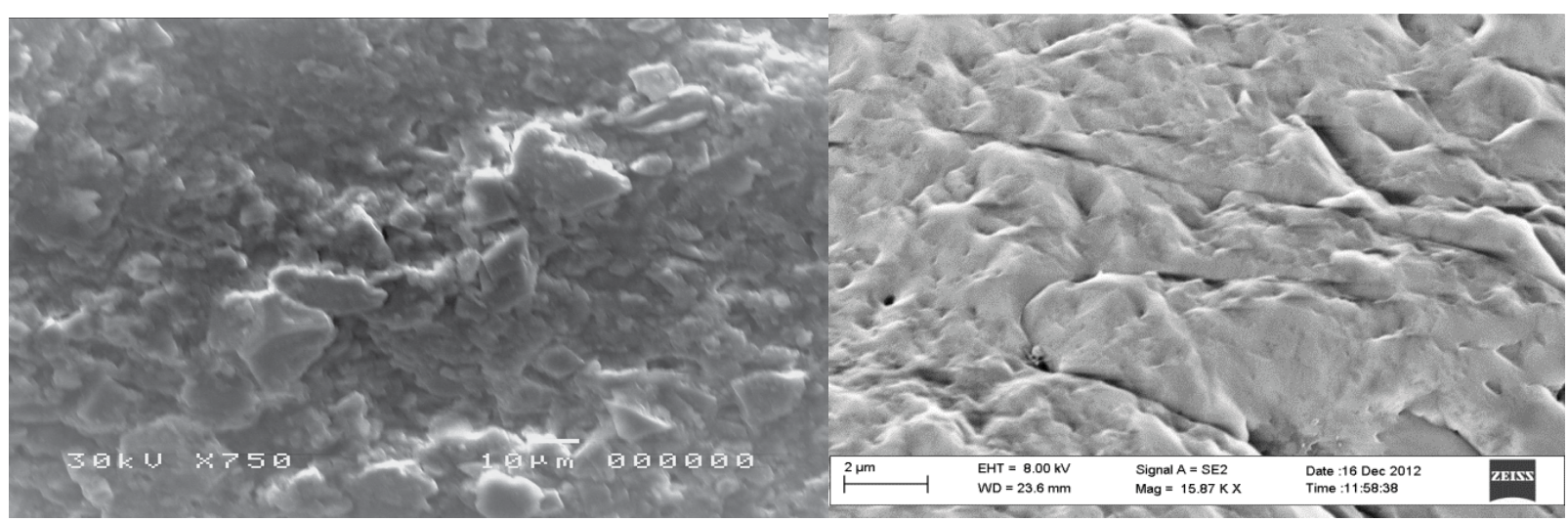

a) Before injection

b) After injection

Figure 6: a) Sandstone sample before injection; b) Sandstone sample after injection. 
can be observed in this pseudo-ternary phase diagram. With the aim of studying the gel strength effects alongside buffers to prevent the $\mathrm{PH}$ value from falling and causing the polymer to breakdown.

\section{Testing in an injection cell}

Rheological experiments were performed in a Brookfield rheometer. The apparent viscosity measurements were obtained by varying shear rate or time, at different temperatures. It was found that, in these experiments, a period of time of $1 \mathrm{~min}$, prior to the actual measurement recording, was necessary in order to allow the system to reach equilibrium (so as to avoid complexities arising from time-dependent phenomena related to gel rheology). Other than both these the samples permeability, porosity, stress, strain and hardness were measured (Table 2).

Tests can be performed to examine the break capacity of the chemical when in contact with oil or formation water. The results were verified by determining the viscosity of the collected samples, after flooding of the smart fluid into the core sample saturated with oil and brine. Samples of fluids were thus collected as a function of time, initially containing oil and brine, and observed under the SEM of the core samples and had its permeability tested.

From the tests, it could be observed that the smart fluid was effective as it performs duliality as both a hydraulic fracture and chemical treatment. The efficiency of the fluid was demonstrated by the primary results from the very first tests. The SEM of the core samples shows that it is more effective with the carbonates rock (Figure 5) under low pressure while its efficiency is higher with sandstone (Figure 6) under high pressure. If used with high concentration with carbonates and with high pressure it can cause a huge fracture in the formation and as it hold its own proppant in nanoscale, it can be a suitable fracture fluid.

From Figure 5 and Figure 6, it was easy to find that, Permeability for both sandstone and Carbonate samples was improving after injection but it was more effective with the carbonates rock (Figure 5) under low pressure while its efficiency is higher with sandstone (Figure 6) under high pressure. If used with high concentration with carbonates and with high pressure it can cause a huge fracture in the formation and as it holds its own proppant in nanoscale, it can be a suitable fracture fluid.

\section{Conclusion}

To simulate reservoir conditions, brine was again injected into the core sample at constant rate, once the reservoirs presented residual oil, in the opposite direction to oil production, at $200 \mathrm{psi}$. The fluid was then injected at a constant flow rate, in the opposite direction to oil production, at $200 \mathrm{psi}$.
Using SEM, it was easy to find that, Permeability for both sandstone and Carbonate samples was improving after injection but it was more effective with the carbonates rock under low pressure while its efficiency is higher with sandstone under high pressure. If used with high concentration with carbonates and with high pressure it can cause a huge fracture in the formation and as it holds its own proppant in nanoscale, it can be a suitable fracture fluid.

Samples were collected, initially with some oil content, these were collected at specifically determined periods of time, starting from the activation of a chronometer when the pressure was applied. After the samples were collected, gel break was verified by assessing the viscosity, in a Brookfield Rheometer, at $26^{\circ} \mathrm{C}$. From the tests, it could be observed that the smart fluid was effective as it performs duality as both a hydraulic fracture and chemical treatment. The efficiency of the fluid was demonstrated by the primary results from several of first tests. The SEM of the core samples shows that it is more effective with the carbonates rock under low pressure while its efficiency is higher with sandstone under high pressure. If we used the high concentration of carbonates and under high pressure it can cause a huge fracture in the formation and as it holds its own proppant in nanoscale, it can be obtain a suitable fracture fluid.

\section{References}

1. Bourgoyne, Adam T (1986) Applied Drilling Engineering. TX: Society of Petroleum Engineers, Richardson.

2. Rahman SS, George V Chilingar (1995) Casing design theory and practice. Amsterdam: Elsevier.

3. I Evdokimov, N Eliseev, A Losev, M Novikov (2006) Emerging petroleum-oriented nanotechnologies for reservoir engineering. SPE Russian Oil and Gas Technical Conference and Exhibition.

4. Md Amanullah, AM Al-Tahini (2009) Nano-Technology - its significance in smart fluid development for oil and gas field application. SPE Saudi Arabia Section Technical Symposium.

5. M Wilson, K Kannangara, G Smith, M Simmons, B Raguse (2002) Nanotechnology, Chapman \& Hall/CRC, Florida.

6. (2013) Bubblers and Cylinders for CVD/ALD Precursor Handling.

7. A Shashurin, M Keidar (2015) Synthesis of $2 D$ materials in arc plasmas. J Phys D Appl Phys 48: 1-18.

8. Techniques for synthesis of nanomaterials (I).

9. McCabe MA, LM Robert, ME Blauch, JM Terracina, LV Lehman, et al. (1999) Investigation of a new fracturing fluid and conductivity enhancement technology on coalbed methane production. SPE mid-continent operations symposium 491-496.

10. Voneiff GW, BM Robinson, SA Holditch (1996) The effects of unbroken fracture fluid on gas well performance. SPE Production \& Facilities 11: 223-229. 